\title{
Analysis of the Psychological Conflict of Contemporary Street Aesthetics in China from the Views of Media Communications
}

\author{
Chen FENG, Hua Wei XU \\ Faculty of Architecture and Urban Planning of Chongqing University, Chongqing, China
}

\begin{abstract}
New media patterns generate more complicated and diversified space forms. Spatial experiences that are instant, fragmented, reality-virtuality interlaced are ubiquitous. Such interlacing brings an enormous impact on traditional Chinese streetscapes, increases the risks of the fragmentation of streetscapes, and leads to the disorder of visual aesthetics. Moreover, it necessarily imposes a great impact on traditional street aesthetics, and causes various psychological conflicts. This study aims to describe this phenomenon and try to explore the reason behind it in new view.
\end{abstract}

\section{Introduction}

The physical environment, electronic information and activities of Chinese urban street spaces in modern times are interacting with each other under the influences of the new media, demonstrating a highly interactive situation, namely, the "mediated quasi-interaction" ${ }^{[1]}$ proposed by John B. Thompson. Physical space is being gradually included as a part of media, acting as a role that disseminates information. Streetscape is enclosed by spatial media of various forms from top to bottom, constructing the appearance of the city with a variety of ways and affecting how people recognize urban space psychologically.

\section{Defamiliarization of Street Visions Caused by Foreign Scenes}

The boundaries of connection in a traditional urban street space place people in a continuous and enclosed realm where the spatial experiences are homogeneous and linear. People's spatial perceptions change along with the patterns and speeds of directions and movements, and people perceive spatial order and structure according to such progressive continuity. The features of visual communication in the age of new media diversify the public interface that was used to be plain, leading to frequent changes of the visual images of street space.

\subsection{The Unconventional "Foreign Landscapes"}

"The worship of differences is built on the loss of differences." ${ }^{[2]}$ With the rapid dissemination of a massive number of image information, disseminators choose to use images with a stronger visual impact while disseminating information in order to prevent them from being devoured. Because visual information that is of easier format and clearer theme can be more easily recognized and accepted in a visual way in terms of avoiding visual fatigue, and it can easily generate visual memory. Architectural forms and street scenes start "disintegrating", bringing an enormous impact on the visual image of street space.

The unconventional "foreign landscapes" are increasingly becoming an integral part of urban public landscapes, breaking the homogeneity of space and forming "singularities" one after another. Endless newfangled buildings seize the eyeballs of pedestrians with their exaggerated and powerful visual appeal. Visual spectacles are now common in street visual images.

\subsection{The Fragmentation and Collage of Streetscapes}

The visual spectacles of streets and surroundings are broken off, splitting the street space, and the saltant image icons seriously damage the connections and integrity of the convey of streetscape traits. Street space is filled with interrupted fragments, fractures and cracks, gradually disproportionating the balance of traditional landscape order, and making the "fragmentation of streetscapes" an increasingly serious problem.

The visual state of being "collage" and disorder make people become used to fragmented thinking. The fission of the mass communication in the urban street public space is now conveying information to smaller groups. The diverse representations in the streets are conducting free collage, and visual methods, such as "incompleteness, fragment, subversion, delusion, absurdity, and eeriness" are like "noisy points in images", breaking off with the surroundings and disproportionating the traditional balance in terms of

\footnotetext{
*Corresponding author: Email: 19535411@qq.com
} 
visual images.

\subsection{The Visual Defamiliarization of Street Space}

We may borrow the term, "noisy point", in electronic imaging, as a way to conclude such phenomenon existed in the visual images of Chinese urban streets. The aesthetic concepts that are harmonious and complete are now being deconstructed and reshaped due to information differentiation of urban street space. The entire image is now disappearing, and those visual orders and rules of previous street space start collapsing, and the streetscapes that were used to be visible to people now no longer have integral clues to be found.

The fragmented thinking caused by such "visual mosaic and excessive symbolic elements", however, covers the differences among symbol vision. The street aesthetics, in general, demonstrate the state of "heteroglossia"[3] and the trend of "visual defamiliarization", quoted Bakhtin. The interface of streets resembles a paper of beautifully written words, which is visible yet unable to interpret the general implication. Foreign landscapes generate powerful visual defamiliarization. Despite of its efforts in innovation and striving for changes, such defamiliarization effects still pose challenges against traditional classic aesthetics. It questions about some of the basic aesthetic principles of urban design, and thoroughly overturns the traditional constituent principle of design, which is "symmetry, proportions, and sequence".

\section{The Tearing of Street Interface Brought by the Trend of Labeling}

Architectural forms may contend for public recognition, stimulate public awareness, and construct the value standard of users with the help of system symbols. Charles Sanders Peirce and Ferdinand de Saussure have proposed a modern academic thought, "symbology", in earlier days. With the use of the basic theories of "symbology", we may regard and interpret modern society in a more clarified way. This provides research basis for us to better understand various symbols.

\subsection{The Unconventional "Foreign Landscapes"}

With the development of new media technologies and modes of transmission, enterprises now have more and more powerful control over visual communications, and their discursive right in terms of space expression has been continuously enhanced. However, spatial visual images with clarified image perception may completely represent unique corporate images, and construct the enterprise's visual image in the city in a labeling way, bringing enormous economic values to the enterprise. Moreover, the commercial architectural interface, which is better in demonstrating hi-tech packaging and the fineness of decoration, becomes the best representation of aesthetic experiences, showing a trend of labeling that is more recognizable and memorable.

Under such influence, designers also believe that "in the construction process of complicated complex of commercial media, a conspicuous information transfer system shall be adopted to replace those inconspicuous ways of express"[4]. Lots of commercial buildings often come across as striking with their supernormal shapes, colors and textures, and they are of intense traits of "sculpture" or "painting", giving people incredible visual association, and becoming "image features" as well as unique "commercial symbols".

\subsection{Exaggerated Symbols that are Different from "Official" Landmarks}

Unlike the "official" background of normal urban landmarks, commercial buildings that are affected by pan-aesthetic orientation are more instantaneous and dramatic. They instinctively cater to the public psychology of cultural consumption, and satisfy people's pursuit of fashion and pleasures. They release the tension of the pursuit of the absolute "official" landmarks. Because their familiar cognitive impression of the public, they achieve a short moment of relaxation psychologically while enhancing visual attraction. Various graphic symbols that are "created" try their uttermost to meet people's pursuit of fashion and pleasures, making them filled with instantaneous and dramatic effects, catering to the public psychology of cultural consumption, quickly occupying our daily vision, and bringing deep "capital branding" on urban public space.

\subsection{The Extreme Simplification of Street Images}

In a chaotic street space where fragmented shapes are scattered around, diverse information symbols are concentrated, and simplistic as well as primitive forms will undoubtedly arouse visual attraction. Under such influence, modern architecture starts pursuing concise "spirit of geometry" again. Streets, as a result, become the arena of commodity devices, symbols and information, and street images quickly become flattened and symbolized.

Concise space images start becoming brand symbols after they have achieved tremendous commercial values, which can be seen from the color tones of buildings to their surfaces, their volumes, and even urban space. Such "labeling" phenomenon of street space has been penetrating ubiquitously, imposing an enormous negative impact on the visual order of urban streets. The labeled buildings resemble symbolic scenes in cities, and bringing negative impacts such as the tearing of urban street interface and the loss of spatial cognition.

\section{The Overload of Street Visual Information Resulted from Image Sticking}

David Shenk discovered that "developed media technologies have brought people from the age where information was insufficient to the age of information 
redundancy"[5]. Excessive hands-down information has been interfering how people estimate and view authentic space environment. People spend lots of time and energies on information processing, and overloading information even makes information anxiety disorder a new issue of the urban development promoted by media.

\subsection{The Explosive Growth of the Total Amount of Street Information}

The simplicity of digital reproduction leads to the explosive growth of the total amount of street information, and people are able to access information of which the amount is many times higher than that in the past in every unit period of time. The life pace of modern society does not allow people to meticulously focus on one object. Therefore, people have to rush through when the street space is filled with overloading visual information, and there is no more viewing of perspective and contemplation. Instead, we have to accept visual objects by means of viewing or glancing. An excessive amount of information leads to the dilemma of information receiving, and we are put in a predicament of insufficient information.

When there is an excessive amount of information, people are interfered so much that they can hardly determine, and their attention becomes the thing being "gnawed". To arouse people's attention better, scenes of stimulation have to be constantly changed in order to promote people's attention shift. Therefore, urban street space has officially entered a space where the competition for attention is extremely fierce.

\subsection{The Collapse of the Hierarchy of Street Information}

Urban streets are gradually evolving into a world of symbols made of information under the influence of new media. Various kinds of information exchange, radiation, spreading and transfer gather here rapidly, and media of communications appear to be highly saturated. The interactive experiences of streetscapes also accelerate the speed of information communications. The openness of structure, the overlapping of functions, and diversity of forms all provide new possibilities for the richness of urban streets. Excessive symbolism and abduction lead to massive waste of urban street space information resources since information is often replaced before it is expressed, and electronic footprints and bitty data collections are scattered everywhere in the street space.

The communications of image information are often ignored and drowned, so epidermidalized method with a strong visual impact is chosen for the expression. This image information that is created in different times is being continuously accumulated on the skins of buildings, gradually leading to overflow and overload. The visual system of streets is manifested as information surplus, overflow and the collapse of information hierarchy.

\subsection{The Influence of Image Saturation on the Delivery of the Street Visual Information}

As the mark of the age of media, overflowing surplus visual information is invading the limited visual space. The rapid information flow of new media has been impacting people's working load limit fiercely. Such surplus information gradually become the so-called "photochemical garbage", generating a sense of pressure on our vision. The bright and noisy urban visual background interfered people's biological rhythms, and affecting the general health levels of people.

The excessive visual image sticking and saturation have completely surpassed the basic reading of the audience towards space sceneries. Various kinds of information are polluting our vision and invading our visual space, thus causing the loss of valuable information, affecting the effective delivery of visual information, causing the fatigue of attention, leading to excessive consumption of attention, and making people unable to extract useful information out of a complicated environment. Such phenomenon of information overload, incapability of filtering and evaluating information is referred to as the "AIDS (anti-information deficiency syndrome)" ${ }^{"[6]}$, according to Boltzmann.

\section{The Space-Time Disorder Resulted from Multi-Dimensional Concatenation}

Various virtual media are creating phantasmagoric reality for cities, and a feeling of space-time tension is generated by the virtual space squeezing the real space. Media arts brought us the idea of "de-distantiation", which eliminates the "aesthetic distance", a point of aesthetic view of authority that has been advocated by traditional aesthetic theories. Media led to the information grafting in the age of globalization, and the random redirection between images that has no inner logics leads to the space-time condensation of street visual scenes.

\subsection{The Dissipation of the Sense of Dimensions of the Street's Real Space-Time}

Urban street space is no longer a pure material and substantial space under the influence of electronic media. Instead, it is changed into a composite state where information elements and virtual space are intermingled, isomorphic co-existing. Therefore, the space boundaries and rules that were used to be well-defined are now promiscuous and confused. "The things that are not supposed to be together and shown to all are now shown." [7]

The "parts and fragments" in street space are collaged, and forms of different histories, cultures and geographies show themselves simultaneously in the same scene, "reflecting the sense of human nature and the historical connotations of the base in the scene" ${ }^{[8]}$ with "real analogs" that extend public memory and feelings. People cannot precisely locate in space dimensions easily, and we are no longer able to determine the environment and define our directions by mobilizing our sensory cognition as we are in it. Gradually, we lost the sense of dimensions of the real space and time. 


\subsection{The Montage-Style Reconstruction of Street Conception Brought by Space-Time Condensation}

Traditional space-time view has gone through fundamental changes in the age of information. The urban street space becomes smaller and smaller, and the interface is no longer continuous and complete. However, the rapid development of science and technology makes the collage of any images easy. As a result, the fragments of city images come in flocks under the background of "space-time condensation", and they change quickly. Street scenes are dissevered into countless non-continuous fragments.

"The non-linear narrative scene" has completely deconstructed and overturned logical narration and time narration. Such "fragmented" scenery fracture facilitates even larger and more zones of fracture in people's daily life like a "butterfly effect". The order between the past, present and future in space becomes confused. Also, the fracture of time and the highlighting of "present" make everything lose their inner connections.

\subsection{The Streetscape Delusion Has Altered the Real Experiences of Space}

The powerful media technologies have altered the way the landscapes are created, allowing the audience to immerse themselves in a world of virtual simulacrums. As a result, the streets are filled with a massive number of exaggerated and implicitly metaphorical "landscape delusion". Such "virtual speed" has breached the boundaries between the real and virtual world, and the extremely surreal world overwhelms us. So many circulating image signals have altered the real experiences of people's urban life. Unknowingly, the recognition of the city's geographical, historical and cultural depth is weakened, and the cultural space is dissevered and overturned.

\section{Conclusion}

This study interprets the aesthetic phenomena of urban streets and aesthetic psychological conflicts, which is different from the studies that traditionally focus on "whether a form is good or bad". It is not limited to the aesthetic study of a certain attribute such as materials, structure and color. Instead, the aesthetic environment and the shift of people's aesthetic psychology are discussed from the perspective of media, and a special study of various psychological conflicts that may take place during the process is carried out.

\section{References}

1. John B. T. Ideology and Modern Culture [M]. Trans. by Xian G. et al. Nanjing: Yilin Press, 2012.

2. Jean B. The Consumer Society $[\mathrm{M}]$. Trans. by Chengfu L., Zhigang Q., Nanjing: Nanjing University Press, 2000.
3. Jean F. L. The Condition of Postmodernity [M]. Trans. by Daozi, Changsha: Hunan Arts Press, 1996.

4. Robert V. Learning from Las Vegas [M]. Trans. by Yifang X., Jian W., China WaterPower Press, 2006.

5. Shenk D. Data Smog: Surviving in the Information Glut [M]. Jiangxi Education Press, 2001.

6. Neil P. Technopoly - The Surrender of Culture to Technology [M]. Trans. by Daokuan H., Peking University Press, 2007.

7. Joshua M. No Sense of Place: The Impact of Electronic Media on Social Behavior [M]. Trans. by Zhijun X. Beijing: Tsinghua University Press, 2002.

8. Colin R. Collage City [M]. Trans. by Tong Ming, Beijing: China Architecture \& Building Press, 2003. 